\title{
Iterative variational mode decomposition and extreme learning machine for gearbox diagnosis based on vibration signals
}

\author{
M. Firdaus Isham ${ }^{1,2^{*}}$, M. Salman Leong ${ }^{1}$ L. M. Hee ${ }^{1}$ and Z. A. B. Ahmad ${ }^{2}$ \\ ${ }^{1}$ Institute of Noise and Vibration, Universiti Teknologi Malaysia, \\ 54100 Kuala Lumpur, Malaysia. \\ ${ }^{2}$ School of Mechanical Engineering, Universiti Teknologi Malaysia, \\ 81310 Skudai, Johor, Malaysia. \\ Phone: +60326154413; Fax: +60326932854 \\ *Email: firdaus060609@gmail.com
}

\begin{abstract}
Vibration-based monitoring and diagnosis provide an excellent and reliable monitoring strategies for maintaining and sustaining a million dollars of industrial assets. The signal processing method is one of the key elements in gearbox fault diagnosis for extracting most useful information from raw vibration signals. Variational mode decomposition (VMD) is one of the recent signal processing methods that helps to solve many limitations in traditional signal processing method. However, pre-determine the input parameters especially the mode number become a challenging task for using this method. Then, this study aims to propose an iterative approach for selecting the mode number for the VMD method by using the normalized mean value (NMV) plot. The NMV value is calculates based on the ratio of a summation of VMD modes and the input signals. The result shows that the proposed iterative VMD approach can select an accurate mode number for the VMD method. Then, the vibration signals decomposed into different VMD modes and used for gearbox fault diagnosis. Statistical features have been extracted from the selected VMD modes and pass into extreme learning machine (ELM) for fault classification. Iterative VMD-ELM provide significance improvement of about $20 \%$ higher accuracy in classification result as compared with EMD-ELM. Hence, this research study offers a new mean for gearbox diagnosis strategy.
\end{abstract}

Keywords: Variational mode decomposition; extreme learning machine; gearbox; signal processing; fault diagnosis; mode selection.

\section{INTRODUCTION}

Gearbox failure is one of the most terrifying problems in many industries. In most cases, the failure may lead to many losses in term of equipment and financial [1-3]. In order to avoid any sort of equipment failures, it is very important to have a good prevention strategy. Vibration is a powerful tool that helps to provide a good information regarding the state of the assets either equipment or structure [4-7]. For machinery monitoring, vibration-based is one of the most popular techniques used in order to maintain and sustain industrial assets due 
to its simple, low-cost and easiness of implementation [8-10]. Therefore, there are many gear monitoring and diagnosis strategies have been proposed over a decade using a lot of different combination of signal processing method and machine learning method in order to achieve better and efficient result [11-15].

The signal processing method is an important element in gearbox diagnosis as it used to extract and provide useful information from the vibration signals. Fast Fourier transform (FFT), Short-Time Fourier transform (STFT), Wigner-Ville distribution (WVD), wavelet transform (WT) and empirical mode decomposition (EMD) are some of the traditional signal processing methods that have been widely used in machine diagnosis. In the last 10 years, the EMD method has been extensively studied due to its capability to solve many limitations for FFT, STFT, WVD and WT [16]. This method has been developed by Huang et al. in 1998 for non-stationary and non-linear data analysis problem where it decomposes signals into different modes called intrinsic mode functions (IMFs) [17]. However, the EMD method suffers from mode mixing and end effect problem on its application in gearbox vibration signals $[18,19]$. Hence, there is a lot of improvement method have been proposed in order to address this limitation for the EMD method. Some of these improvement methods are ensemble EMD (EEMD) [20], Complementary EEMD (CEEMD) [21], partly EEMD (EEMD) [22], local mean decomposition (LMD) [23] and intrinsic time-scale decomposition (ITD) [24].

Recently, another method for solving the limitation of the EMD method has been proposed by Dragomiretskiy and Zosso in 2014 which is the variational mode decomposition (VMD) [25]. The VMD method decomposed signals into different modes known as variational mode function (VMFs) by adopting the unique decomposition procedure, alternate direction method of multiplier (ADMM) which replaced traditional sifting process used in the EMD method [26]. This makes the VMD method capable to provide a good solution in decomposing the vibration signals by solving a mode mixing problem and restrain an end effect problem [27-29]. The VMD method required the input parameters especially the mode number to be well-defined in order to have a good decomposition result. Hence, selecting the mode number become an open research problem for the VMD method [30]. In the previous study, selecting the mode number for the VMD method is based on the spectrum peaks that makes it difficult to obtain a satisfactory analysis result [26,31]. Therefore, some solution for selecting the mode number has been proposed recently by $\mathrm{Li}$ et al. by selecting the mode number based on envelope spectrum peaks using locally weighted scatterplot smoothing (LOWESS) [30] and Zhang et al. using correlation and energy ratio plot to select the mode number [32].

Therefore, this research study aims to propose an iterative VMD approach for selecting the mode number for the VMD method by using the normalized mean value (NMV) plot. The NMV value is calculates based on the ratio between the summation of VMFs and the input signals which adopt the similarities concept between the summation of VMFs and its original signals. The mode number is selected based on the first point reached the steady state condition in the NMV plot. For validation, three simulated signals have been used as discussed in this paper. Statistical parameters have been used as features for extreme learning machine (ELM) algorithm for fault classification. An online wind turbine gearbox vibration signals consist of healthy and faulty datasets have been used in this paper for the application of fault diagnosis approach based on VMD-ELM in gearbox applications. For comparison, EMD-ELM method has been used in this paper. This research study provides a new idea on 
selecting the mode number for the VMD method by using the NMV plot and its application with ELM algorithm for gearbox diagnosis.

The rest of the paper is organized as follows. In section 2, the basic idea of the VMD method and extreme learning machine algorithm have been presented. Section 3, introduces the proposed method and section 4 present the result of the proposed method and diagnosis process for gearbox using VMD and ELM methods.

\section{THEORY AND METHODOLOGY}

\section{Variational Mode Decomposition}

The VMD method consists of three basic concepts which are Wiener filtering, heterodyne demodulation, and one-dimensional Hilbert transform as discussed by Dragomeritskiy and Zosso in their paper [25]. This method decomposed vibration signals into sets of sub-signals called variational mode function (VMF), and also define as amplitude-modulated and frequency-modulated (AM-FM) signals. In order to run the VMD algorithm, there are five parameters need to be pre-determined which are mode number, balancing parameter, initial omega, time-step of dual ascent and tolerance. The full algorithm for the VMD method is given as the following expression.

Step 1: Initialize $\left\{\hat{u}_{k}^{1}\right\},\left\{\hat{u}_{k}^{1}\right\}, \lambda^{1}, n \leftarrow 0$.

Step 2: The value of $u_{k}, \omega_{k}$, and $\lambda$ is updated according to the following formula:

$$
\begin{gathered}
\hat{u}_{k}^{n+1} \leftarrow \frac{\hat{f}(\omega)-\sum_{i<k} \hat{u}_{i}^{n+1}(\omega)-\sum_{i>k} \hat{u}_{i}^{n}(\omega)+\frac{\widehat{\lambda}^{n}(\omega)}{2}}{1+2 \alpha\left(\omega-\omega_{k}^{n}\right)^{2}} \\
\omega_{k}^{n+1} \leftarrow \frac{\int_{0}^{+\infty} \omega\left|\hat{u}_{k}^{n+1}(\omega)\right|^{2} d \omega}{\int_{0}^{+\infty}\left|\hat{u}_{k}^{n+1}(\omega)\right|^{2} d \omega} \\
\hat{\lambda}^{n+1}(\omega) \leftarrow \hat{\lambda}^{n}(\omega)+\tau\left[\hat{f}(\omega)-\sum_{k} \hat{u}_{k}^{n+1}(\omega)\right]
\end{gathered}
$$

Step 3: Repeat the iterative process from 2 until the function is converge based on convergence criteria satisfied the condition of $\sum_{k}\left\|\hat{u}_{k}^{n+1}-\hat{u}_{k}^{n}\right\|_{2}^{2} /\left\|\hat{u}_{k}^{n}\right\|_{2}^{2}<\epsilon$, where $\epsilon$ is a given accuracy requirement.

\section{Extreme Learning Machine}

Extreme Learning machine (ELM) algorithm is a simple and effective algorithm for training single-hidden layer feedforward neural network (SLFNs) as proposed by Huang et al. [33]. Basically, SLFNs consists of the input layer ( $n$ neurons correspond to $n$ input variables), a hidden layer ( $I$ neurons) and an output layer ( $m$ neurons correspond to $m$ output variables). The optimal solution by using the ELM algorithm can be achieved by adjusting the number of hidden layer neurons. The ELM algorithm can provide fast learning process and helps to solve issues on conventional gradient-based algorithm for SLFNs [33]. The ELM algorithm given as the following description. 
Given a training dataset $\left\{\left(x_{i}, y_{i}\right), i=1,2, \ldots, N\right\}$, where $x_{i}=\left[x_{i 1}, x_{i 2}, \ldots, x_{i n}\right]^{T} \in R$ is the input of the sample and $y_{i}=\left[y_{i 1}, y_{i 2}, \ldots, y_{i n}\right]^{T} \in R$ is an expected output of the sample. Activation function in the hidden layer of the network is $g(x)$ and the output of SLFNs is given by the following Equation (4):

$$
o_{i}=\sum_{j=1}^{L} \beta_{j} G\left(a_{j}, b_{j}, x_{j}\right)
$$

where $L$ is the number of hidden nodes, $a_{j}$ and $b_{j}$ are the $j$ th hidden node's parameters assigned in random, $\beta_{j}=R$ is the output weight vector with SLFN output of the matrix can be expressed as below Equation (5):

$$
O=H \beta
$$

where $H$ is a hidden layer output matrix of the network, $H_{i j}$ is a $j$ th hidden node with respect to input samples of $x_{i} \cdot \beta=\left[\beta_{1}, \beta_{2}, \ldots . \beta_{L}\right]^{T}$ and $O=\left[o_{1}, o_{2}, \ldots . o_{L}\right]^{T}$. Then, the objective function of ELM can be expressed as below Equation (6):

$$
\beta=H^{+} Y=\left(H^{T} H\right)^{-1} H^{T} Y
$$

where $H^{+}$is a Moore-Penrose generalized inverse of matrix $H$ and $Y=\left[y_{1}, y_{2}, \ldots, y_{n}\right]^{T}$. The above output weight matrix $\beta$ minimizes the cost function $\|O-Y\|$.

\section{Iterative VMD Methodology}

Selecting an accurate mode number is very important for the VMD method in order to ensure the accuracy of the decomposition result and the performance of the VMD method in the diagnosis process. The proposed iterative VMD using the NMV plot is presented in this section. In mathematics, the mean value is the average or central value for the sets of number. In vibration signals, the mean value represents the central value line for the signals. The NMV value is the mean ratio value between the summation of VMFs and its original signals. The NMV value is calculated based on Equation (7). The NMV plot is the plot of NMV value against the mode number. The first point to reach steady state in the NMV plot is selected as the mode number. This is based on the similarity concept theory between VMFs and its original signals. When a signals is decomposed into sets of VMFs, the summation or combination of VMFs should have the same characteristic with the original signals where NMV is used to calculate this similarity. The good NMV value should be equal to 1 for clean signals or approaching 1 for a noisy signals. The whole framework of the proposed iterative VMD method with NMV plot is shown in Figure 1. 


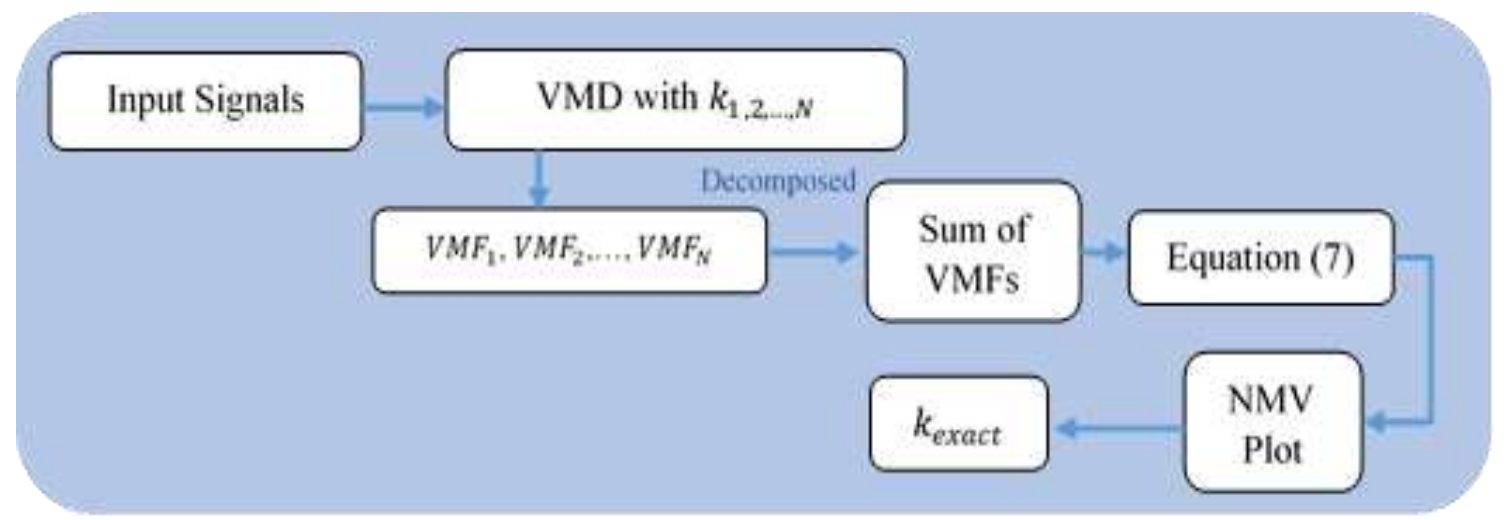

Figure 1. Proposed iterative VMD with NMV plot.

$$
M V=\frac{\left(\frac{\sum_{i=1}^{n} x_{v m f, i}}{n}\right)}{\left(\frac{\sum_{i=1}^{n} x_{\text {original }, i}}{n}\right)}
$$

In order to validate the approach in selecting the mode number, three simulated signals have been used as described in Equation (8a) - (8d) and Figure 2. Then, this simulated signal is passed into the proposed iterative VMD method as shown in Figure 1 which consists of 5 procedure which is (i) decompose signals into sets of VMFs with different mode number, (ii) summation of VMFs, (iii) Calculate NMV value, (iv) NMV plotting and (v) mode number selection based on NMV plot. For the first procedure, the maximum number of modes for this simulated signals is set to 10 . This value is set based on the assumption and the suggested maximum mode number value for gearbox application is 15 or 20. Figure 3 shows the NMV plot for the simulated signals. Based on the plot, the first point reaches steady state is 3 . Hence, the exact mode number for the simulated signals is 3 as the result shown in Figure 5. Figure 4 and 6 shows the decomposition result when the mode number is 2 and 4 in order to shows the performance of the VMD method with an inaccurate mode number. This will caused the under-decomposed problem and over-decomposed problem where the signal is not reconstructed properly which may cause the information loss in a further stage of analysis.

$$
\begin{gathered}
x_{1}(t)=3 \sin (2 \pi 5 t) \\
x_{2}(t)=0.4 \sin (2 \pi 200 t) \\
x_{3}(t)=1.2 \sin (2 \pi 50 t) \\
X(t)=x_{1}(t)+x_{2}(t)+x_{3}(t)
\end{gathered}
$$



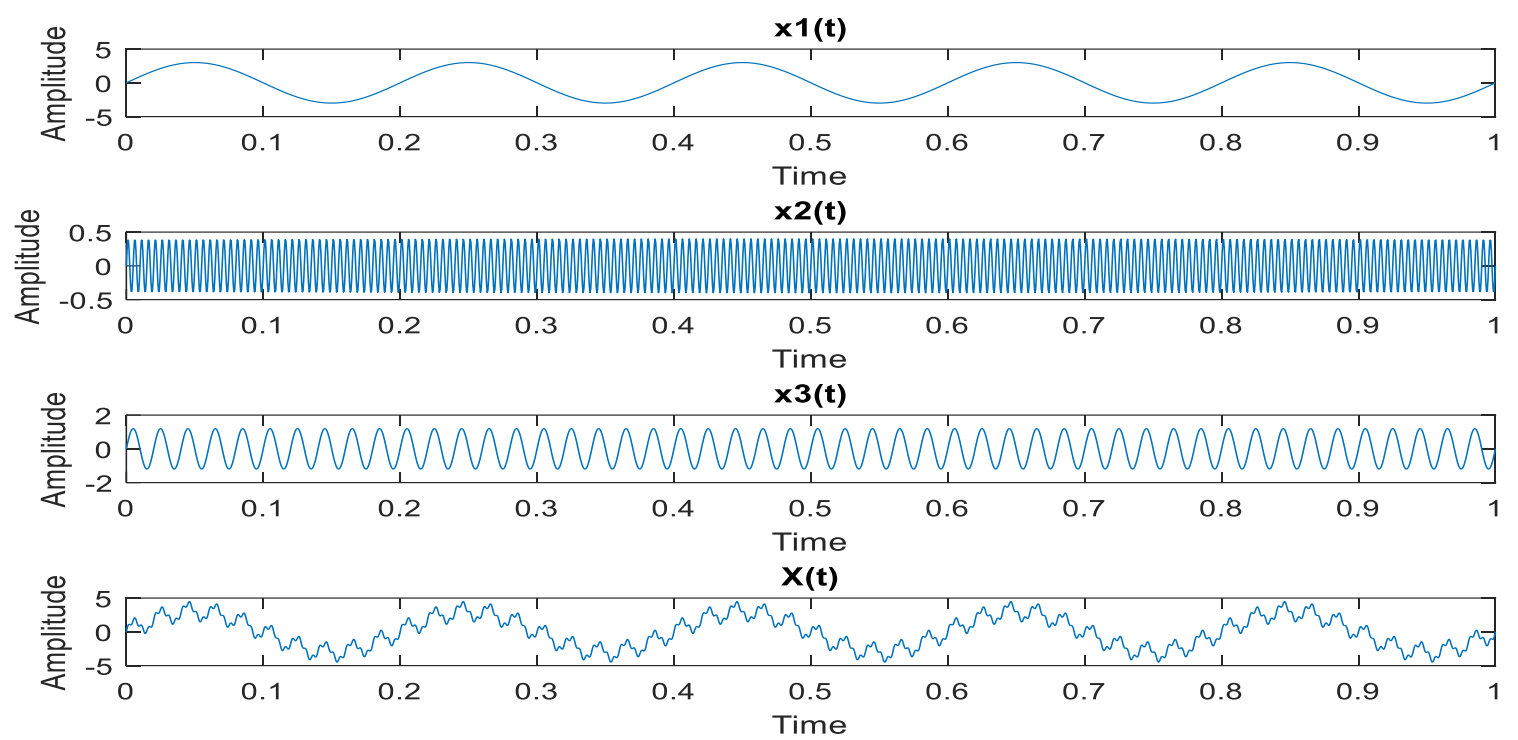

Figure 2. Simulated signals.

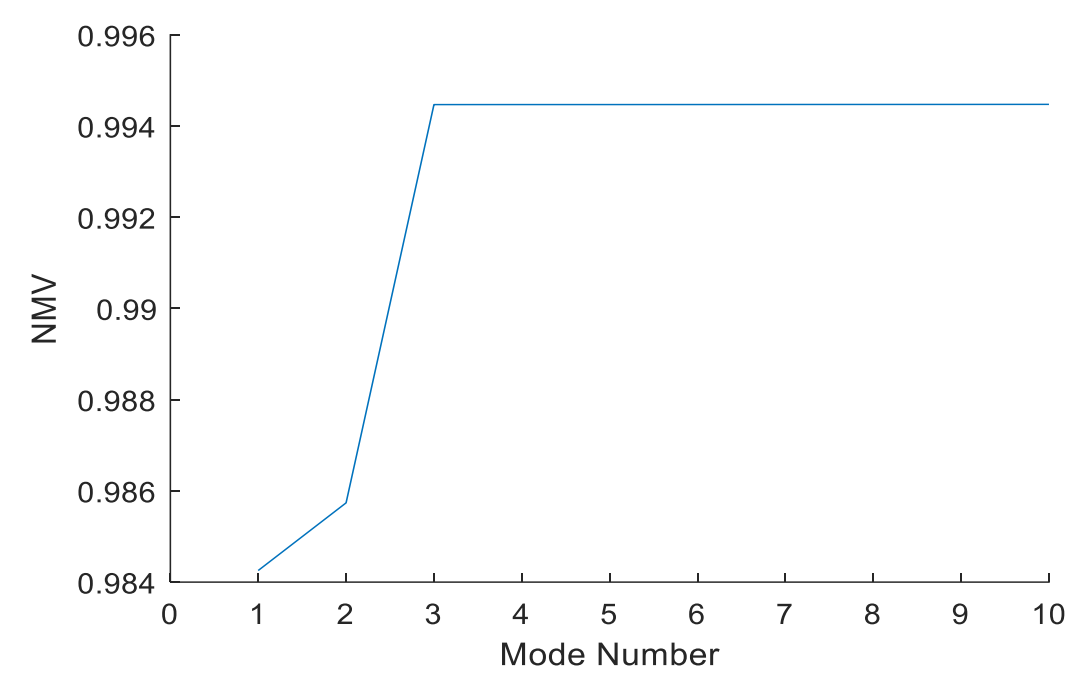

Figure 3. NMV Plot for simulated signals.
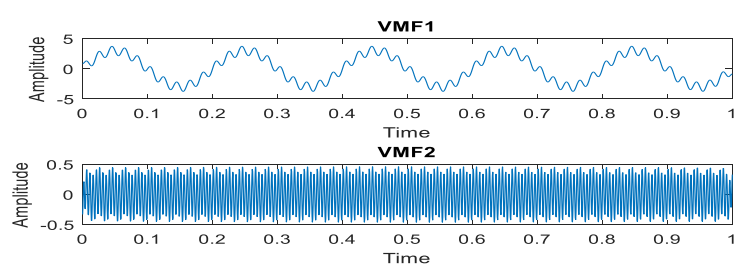

Figure 4. VMD decomposition result when the mode number is 2 . 


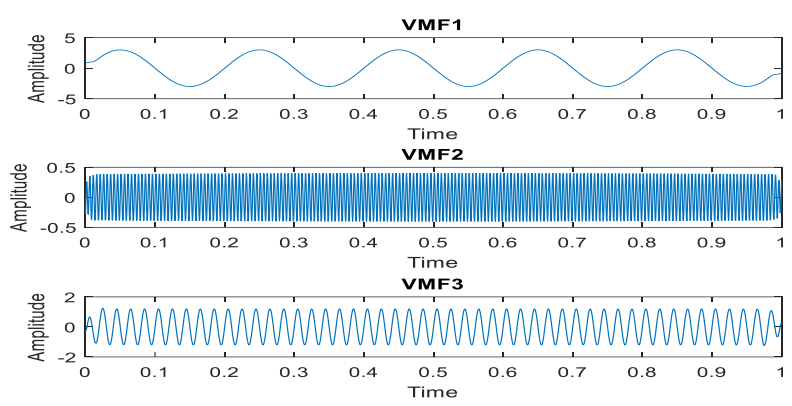

Figure 5. VMD decomposition result when the mode number is 3.
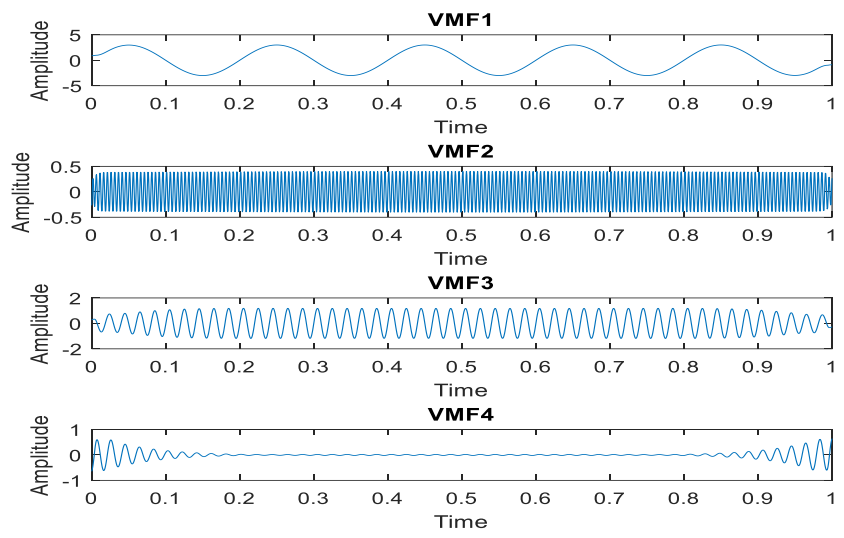

Figure 6. VMD decomposition result when the mode number is 4.

\section{Iterative VMD-ELM for Gearbox Fault Diagnosis Methodology}

Iterative VMD and ELM approach for gearbox fault diagnosis is discussed in this section. An online wind turbine gearbox vibration signals consist of healthy and faulty signals are used in this paper. The signals are downloaded from Acoustic and Vibration database provided by Bechhoefer [34]. The vibration signals are taken from Vestas V90 Hansen gearbox with a power rating of $3 \mathrm{MW}$ and the nominal speed of $30 \mathrm{~Hz}$. The sampling rate is $97656 \mathrm{~Hz}$, record length of 6 seconds, radial direction using the accelerometer sensor. The faulty condition shown in Figure 7. 


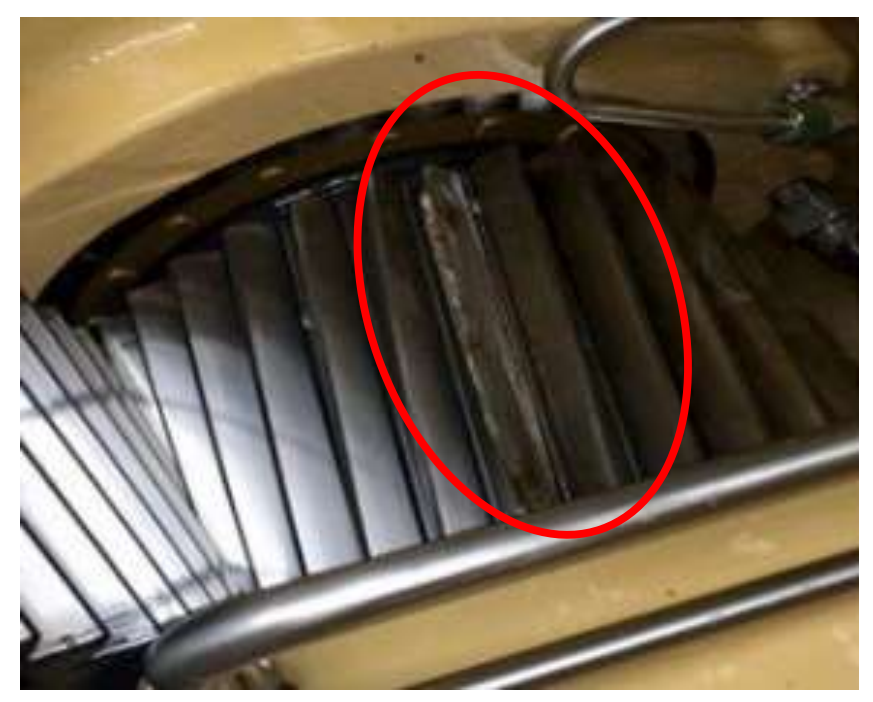

Figure 7. Wear on the gearbox.

Recent gearbox diagnosis method mainly consists of signal processing, features extraction, features selection and fault classification. For this paper, it consists of signal processing using VMD method, features extraction using statistical features and fault classification using ELM. The complete procedure of iterative VMD and ELM for gearbox fault diagnosis can be described as follows.

i) Separate signal samples based on one revolution, $x_{h 1}, x_{h 2}, \ldots, x_{h 20}$ and $x_{f 1}, x_{f 2}, \ldots, x_{f 20}$.

ii) Select an accurate mode number for each signal samples using iterative VMD method, $k_{x_{h 1}}, k_{x_{h 2}}, \ldots, k_{x_{h 20}}$ and $k_{x_{f 1}}, k_{x_{f 2}}, \ldots, k_{x_{f 20}}$.

iii) Decomposed each signal samples into sets of VMFs using the selected mode number.

iv) Select significance VMFs.

v) Extract statistical parameter features from each VMFs.

vi) Training and testing using ELM for fault classification.

For this research, there are 120 signal samples consists of 60 signal samples from healthy datasets and 60 signal samples from faulty datasets are used. Then, the procedure i, ii and iii are proceeded for each signal samples using the iterative VMD with NMV plot in order to have an accurate decomposition result for each signal samples. In procedure iv, the significance VMFs are selected based on its frequency characteristic of $1 \mathrm{X}$ Gear Mesh Frequency (GMF), 2 X GMF and 3 X GMF. Hence, VMF1, VMF2, VMF3, and VMF4 are selected as significance VMFs for each signal samples. Then, statistical features listed in Table 1 are extracted from each VMFs for each signal samples. Hence, there will be 16 features samples for each signal samples and total features for all the signal samples is 1920 features. Table 2 summarized the details on the distribution of training and testing features for ELM classification. Then, the ELM is run for fault classification for healthy and faulty gearbox condition. 
Table 1. List of statistical features used.

\begin{tabular}{lc}
\hline Statistical Parameter & Equation \\
\hline Crest factor & $\frac{\max |x(n)|}{\sqrt{\frac{1}{N} \sum_{n=1}^{N} x(n)^{2}}}$ \\
Shape factor & $\frac{\sqrt{\frac{1}{N} \sum_{n=1}^{N} x(n)^{2}}}{\frac{1}{N} \sum_{n=1}^{N}|x(n)|}$ \\
Impulse factor & $\frac{\max |x(n)|}{\frac{1}{N} \sum_{n=1}^{N}|x(n)|}$ \\
Margin factor & $\frac{\max |x(n)|}{\left(\frac{1}{N} \sum_{n=1}^{N} \sqrt{|x(n)|}\right)^{2}}$ \\
\hline
\end{tabular}

Table 2. Training and testing samples detail for ELM.

\begin{tabular}{lcclc}
\hline Algorithm & $\begin{array}{c}\text { Training } \\
\text { Sample }\end{array}$ & $\begin{array}{c}\text { Testing } \\
\text { Sample }\end{array}$ & Condition & Label \\
\hline ELM & $40 \times 16$ & $20 \times 16$ & Healthy & 0 \\
& $40 \times 16$ & $20 \times 16$ & Faulty & 1 \\
EMD & $40 \times 16$ & $20 \times 16$ & Healthy & 0 \\
& $40 \times 16$ & $20 \times 16$ & Faulty & 1 \\
\hline
\end{tabular}

\section{RESULTS AND DISCUSSION}

\section{Selected Mode for Gearbox Vibration Signals}

As mention earlier, selecting the mode number is very important to have a good decomposition accuracy and good performance when using the VMD method. All 120 signal samples have been passed into the iterative VMD method in order to have an accurate mode number for each signal samples and the result are shown in Figure 8. The healthy signals have less mode number as compared with the faulty signals as expected due to the different of complexness between healthy and faulty signals. One healthy signal, $x_{h 1}$ and one faulty signal, $x_{f 1}$ are extracted from the result in order to demonstrate the selection process. The signals are shown in Figure 9 with its NMV plot shown in Figure 10. Based on this plot, the steady state point can be clearly seen as $x_{h 1}$ has 11 modes and $x_{f 1}$ has 12 modes. The selection is based on the first point reach steady state within the NMV plot. This is the point where the signals characteristic between summation of the VMFs and its original signals are reaching similarity and beyond this first point, the value will be only steady state and approaching one. Selecting the mode number less or higher than this first value is not recommended as it will cause a problem known as under-decomposed and over-decomposed 
problem to occur in the decomposition result. Under-decomposed problem is defined where the two modes mixed together in a single VMF whereas the over-decomposed defined as a single mode is separated into two VMF. Both problems will cause a significance impact on the diagnosis result.

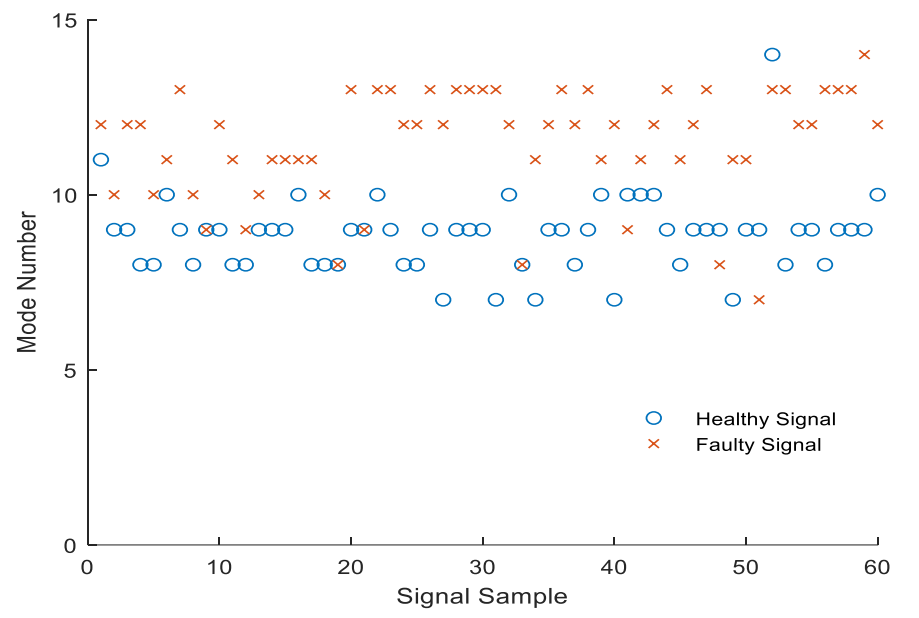

Figure 8. Selected mode number for each signal samples using iterative VMD method.
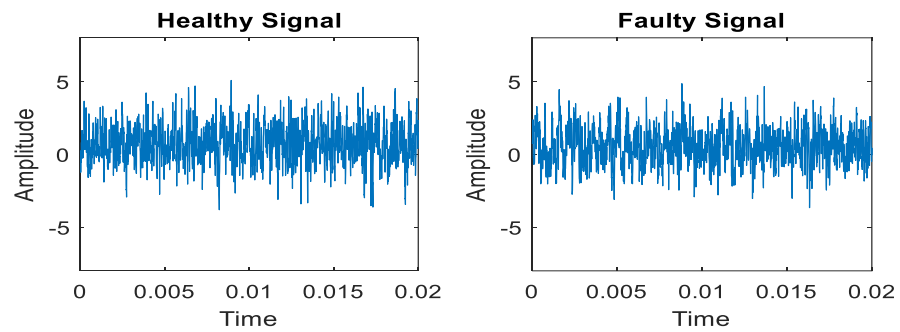

Figure 9. Healthy and faulty gearbox vibration signals.

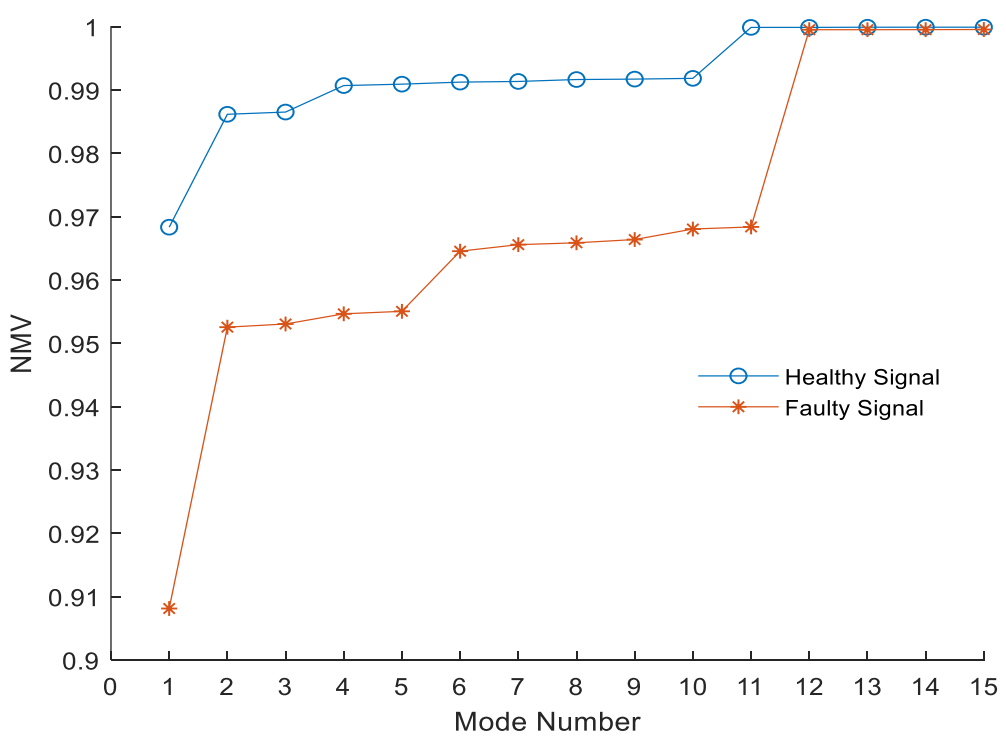

Figure 10. NMV plot for healthy and faulty signals. 


\section{Gearbox Diagnosis}

Gearbox diagnosis is a good technique in order to maintain and sustain the gearbox unit. It also helps to provide a necessary information regarding the condition of the gearbox unit. In this study, the classification is only divided into two state which is a healthy and faulty state. The training dataset and testing dataset described in Table 2 are used with ELM for classification. Figure 11 shows the structure of ELM which consists of an input layer, singlehidden neurons and the output layer.

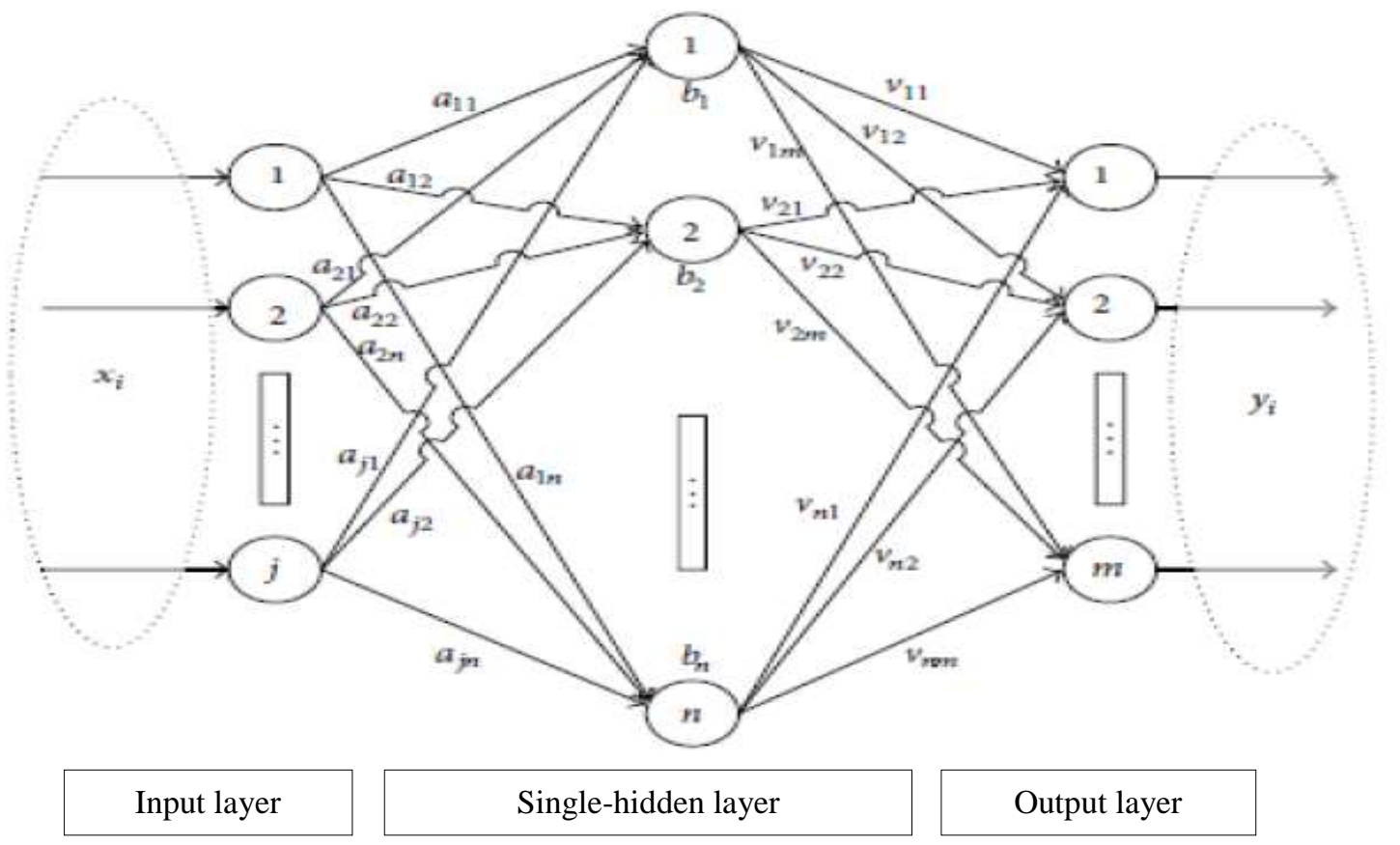

Figure 11. Structure of ELM.

All the features from each signals sample are pass into ELM algorithm and the result is shown in Table 3. The result has been compared with the EMD-ELM method. The overall classification result is calculated based on the average classification value of $\mathrm{A}, \mathrm{B}$, and $\mathrm{C}$ of training and testing performance. A defines as the average performance between 1 to 200 hidden neurons number set in ELM, B defines as the average performance when the hidden neurons is set to 16 and run for 30 times in order to avoid tweak problem [35], and C defines as the average performance when the hidden neurons number is set to 16 . The hidden neurons number is set to 16 due to it used 16 different features for each signal samples. The same approach is used for the EMD-ELM but the hidden neurons number is set to 4 instead of 16 due to 4 different of features used.

Iterative VMD-ELM provides a significant performance with an overall classification rate of $88 \%$, which is $20 \%$ higher than EMD-ELM with $68 \%$. This shows that the iterative VMD method is capable to provide a good signals processing result for the gearbox vibration signals by providing an accurate decomposition result. With a good decomposition result, it helps to extract the most information from the gearbox vibration signals and also helps to reduce the noise within the signal [36-38]. Besides that, good decomposition result also helps to provide better features value for ELM. The fundamental decomposition theory makes VMD provided a better result from EMD. As mention earlier, VMD using ADMM instead 
of sifting process in EMD. Basically, VMD decomposition process is done in the frequency domain based on mode centre frequency whereas the EMD sifting process is done using an enveloping time series signal [17,25]. Sifting process method is a sensitive method for a noisy signals due to the enveloping process miss some important point during the envelope process $[39,40]$. This makes the EMD method suffers in decomposing the gearbox vibration signals whereas the VMD method is capable to do it by providing the accurate mode number using the NVM plot. Therefore, the signal processing method is very important in gearbox diagnosis order to have a diagnosis performance.

However, the performance of iterative VMD-ELM still can be improved for future study as $88 \%$ of the classification rate is still far from $100 \%$. There are many factors influencing the classification rate such as the sensitivity of features, ELM parameters and the ELM training and testing performance. Features are the most important element in machine learning algorithm as it makes the machine learning algorithm to "see" based on its value as the healthy signals basically will give different value compared with the faulty signals. Hence, features selection or scaling may help to improve the classification rate [41]. To run ELM, some parameter need to be set such as its kernel function, bias, and the weight value. In this study, the standard RBF kernel function is used and random value for bias and weight is applied. Using better kernel function such as RBF-UKF kernel function proposed by Chen et al. and optimizing the bias and weight value using a heuristic algorithm such as genetic algorithm (GA), particle swarm optimization (PSO) and differential evolution (DE) may help to improve the classification rate [42-45]. Besides that, the training phase in ELM is very important in order to have a better testing result. The distribution of training always need to be higher than training data as the more the machine has been trained, the more sensitive the machine toward the features value which make it produce better classification result.

Table 3. Gearbox diagnosis result using ELM algorithm.

\begin{tabular}{|c|c|c|c|c|c|c|c|}
\hline \multirow[t]{2}{*}{ Method } & \multicolumn{3}{|c|}{$\begin{array}{c}\text { Training Performance } \\
(\%)\end{array}$} & \multicolumn{3}{|c|}{$\begin{array}{c}\text { Testing Performance } \\
(\%)\end{array}$} & $\begin{array}{l}\text { Overall } \\
(\%)\end{array}$ \\
\hline & $\mathrm{A}$ & B & $\mathrm{C}$ & $\mathrm{A}$ & $\mathrm{B}$ & $\mathrm{C}$ & All \\
\hline VMD-ELM & 98.97 & 94.75 & 70.00 & 85.94 & 96.58 & 82.50 & 88.12 \\
\hline EMD-ELM & 92.34 & 62.17 & 63.75 & 55.53 & 64.00 & 72.50 & 68.28 \\
\hline
\end{tabular}

\section{CONCLUSIONS}

Gearbox unit is a key component in a most industrial application such as wind turbine, gas turbine, and aviation and automotive. In recent years, the accuracy and effectiveness of gearbox diagnosis strategies have been paid more attention by researchers and experts. Efficient and effective gearbox diagnosis result have a significant impact on the most industry. In this paper, a mode selection method using iterative VMD with NMV plot is proposed and used with ELM for gearbox diagnosis strategies. Online wind turbine gearbox vibration signals downloaded from Acoustics and Vibration Database have been used. The proposed iterative VMD provide a good solution in selecting the mode number as it shows a significant performance in determining the exact mode number for simulated signals and 
gearbox vibration signals. Iterative VMD-ELM provides a significant result for fault classification as compared with EMD-ELM in this study. This shows that the proposed iterative VMD manage to provide an accurate decomposition result for the VMD method. Besides that, VMD method with accurate decomposition result produced good features sensitivity for ELM algorithm. With these capabilities, the iterative VMD-ELM can provide efficient and effective gearbox diagnosis strategy for industrial applications. Even though there are a lot of gearbox diagnosis strategy has been proposed, the approach for each proposed strategy may be different with one another in terms of signal processing, features extraction, features selection and machine learning. In this paper, iterative VMD with NMV plots and ELM can contribute for efficient and effective gearbox diagnosis strategies. Some of the improvement also have been discussed in this paper for future improvement of iterative VMD-ELM. In the future, this method also will be applied for other machinery application such as bearing, shaft and belt to validate the performance and superiority of this method.

\section{ACKNOWLEDGMENTS}

The authors would like to extend their greatest gratitude to the Institute of Noise and Vibration UTM for funding the study under the Higher Institution Centre of Excellence (HICoE) Grant Scheme (R.K130000.7809.4J225). Additional funding for this research also comes from the UTM Research University Grant (Q.K130000.2543.11H36), and Fundamental Research Grant Scheme (R.K130000.7840.4F653) by The Ministry of Higher Education Malaysia.

\section{REFERENCES}

[1] Ulus S, Erkaya S. An experimental study on gear diagnosis by using acoustic emission technique. International Journal of Acoustics and Vibration 2016;21:10311.

[2] Chen J, Li Z, Pan J, Chen G, Zi Y, Yuan J, Chen B, He Z. Wavelet transform based on inner product in fault diagnosis of rotating machinery: A review. Mechanical Systems and Signal Processing 2016;70-71:1-35.

[3] Hanapi S, Fakharuzi MHAM, Rahim AHA, Tijani AS, Sainan KI, Mohamed WANW. Effect of gear ratio on the dc motor efficiency of a mini- fuel-cell vehicle cruising at constant speeds. Journal of Mechanical Engineering and Sciences 2015;8(6):1460-1471.

[4] Mohamad N, Yaakub J, Maulod HEA, Jeefferie AR, Yuhazri MY, Lau K, Ahsan Q, Shueb M, Othman R. Vibrational damping behaviors of graphene nanoplatelets reinforced NR/EPDM nanocomposites. Journal of Mechanical Engineering and Sciences 2017;11(4):3274-3287.

[5] Hussin WNW, Harun FN, Mohd MH, Rahman MAA. Analytical modelling prediction by using wake oscillator model for vortex-induced vibrations. Journal of Mechanical Engineering and Sciences 2017;11(4):3116-3128.

[6] Rahman N, Alam MN, Junaid M. Active vibration control of composite shallow shells: An integrated approach. Journal of Mechanical Engineering and Sciences. 2018;12(1):3354-3369. 
[7] Isham MF, Leong MS, Lim MH, Ahmad ZA. Variational mode decomposition for rotating machinery condition monitoring using vibration signals. Transactions of Nanjing University of Aeronautics and Astronautics 2018;35(1):38-50.

[8] Al-Badour F, Sunar M, Cheded L. Vibration analysis of rotating machinery using time-frequency analysis and wavelet techniques. Mechanical Systems and Signal Processing 2011;25(6):2083-2101.

[9] Deng L, Zhao R. A vibration analysis method based on hybrid techniques and its application to rotating machinery. Measurement 2013;46(9):3671-3682.

[10] YanPing Z, ShuHong H, JingHong H, Tao S, Wei L. Continuous wavelet grey moment approach for vibration analysis of rotating machinery. Mechanical Systems and Signal Processing 2006;20(5):1202-1220.

[11] Attoui I, Boudiaf A, Fergani N, Oudjani B, Boutasseta N, Deliou A. Vibration-based gearbox fault diagnosis by DWPT and PCA approaches and an adaptive neuro-fuzzy inference system. International Conference on Sciences and Techniques of Automatic Control and Computer Engineering 2015:234-239.

[12] Dabrowski D. Condition monitoring of planetary gearbox by hardware implementation of artificial neural networks. Measurement: Journal of the International Measurement Confederation 2016;91:295-308.

[13] Chen Z, Li C, Sanchez R. Gearbox fault identification and classification with convolutional neural networks. Shock and Vibration 2015;2015.

[14] Er-raoudi M, Diany M, Aissaoui H, Mabrouki M. Gear fault detection using artificial neural networks with discrete wavelet transform and principal component analysis. Journal of Mechanical Engineering and Sciences 2016;10(2):2016-2029.

[15] Saufi SR, Ahmad ZAB, Leong MS, Lim MH. Differential evolution optimization for resilient stacked sparse autoencoder and its applications on bearing fault diagnosis. Measurement Science and Technology 2018;29(12):125002.

[16] Isham MF, Leong MS, Hee LM, Ahmad ZAB. Empirical mode decomposition: A review on mode selection method for rotating machinery diagnosis. International Journal of Mechanical Engineering and Technology 2017;8(6):16-26.

[17] Huang N, Shen Z, Long S, Wu M, Shih H, Zheng Q, Yen N, Tung C, Liu H. The empirical mode decomposition and the Hilbert spectrum for nonlinear and nonstationary time series analysis. Proceedings of the Royal Society A: Mathematical, Physical and Engineering Sciences. 1998;454(1971):995,903.

[18] Chen G, Wang Z. A signal decomposition theorem with Hilbert transform and its application to narrowband time series with closely spaced frequency components. Mechanical Systems and Signal Processing 2012;28:258-279.

[19] Rato RT, Ortigueira MD, Batista AG. On the HHT, its problems, and some solutions. Mechanical Systems and Signal Processing 2008;22(6):1374-1394.

[20] Huang NE, Wu Z. Ensemble Empirical Mode Decomposition: a Noise-Assisted Data Analysis Method. Advances in Adaptive Data Analysis. 2009;1.

[21] Yeh JR, Shieh JS. Complementary ensemble empirical mode decomposition: A novel enhanced data analysis method. Advances in Adaptive Data Analysis 2010;2(2):135156.

[22] Zheng J, Cheng J, Yang Y. Partly ensemble empirical mode decomposition: An improved noise-assisted method for eliminating mode mixing. Signal Processing 2014;96:362-274. 
[23] Smith JS. The local mean decomposition and its application to EEG perception data. Journal of The Royal Society Interface 2005;2(5):443-454.

[24] Frei MG, Osorio I. Intrinsic time-scale decomposition : time - frequency - energy analysis and real-time filtering of non-stationary signals. Proceedings of The Royal Society A 2007:321-342.

[25] Dragomiretskiy K, Zosso D. Variational Mode Decomposition. IEEE Transactions on Signal Processing 2014;62(3):531-544.

[26] Mohanty S, Gupta KK, Raju KS. Comparative study between VMD and EMD in bearing fault diagnosis. International Conference on Industrial and Information Systems 2014:1-6.

[27] Mahgoun H, Chaari F, Felkaoui A. Detection of gear faults in variable rotating speed using variational mode decomposition (VMD). Mechanics \& Industry 2016;17(2).

[28] Muralidharan A, Sugumaran V, Soman KP, Amarnath M. Fault diagnosis of helical gear box using variational mode decomposition and random forest algorithm. SDHM Structural Durability and Health Monitoring 2015;10(1):55-80.

[29] Isham MF, Leong MS, Lim MH, Ahmad ZA. Variational mode decomposition: mode determination method for rotating machinery diagnosis. Journal of Vibroengineering 2018;20(7):2604-2621.

[30] Li Z, Chen J, Zi Y, Pan J. Independence-oriented VMD to identify fault feature for wheel set bearing fault diagnosis of high speed locomotive. Mechanical Systems and Signal Processing 2017;85:512-529.

[31] Gupta KK, Raju KS. Bearing fault analysis using variational mode decomposition. International Conference on Industrial and Information Systems 2014:1-6.

[32] Zhang M, Jiang Z, Feng K. Research on variational mode decomposition in rolling bearings fault diagnosis of the multistage centrifugal pump. Mechanical Systems and Signal Processing 2017;93:460-493.

[33] Huang GB, Zhu QY, Siew CK. Extreme learning machine: Theory and applications. Neurocomputing 2006;70(1-3):489-501.

[34] Bechhoefer E. High speed gear dataset. Acoustics and Vibration Database. 2014.

[35] Tong Q, Cao J, Han B, Zhang X, Nie Z, Wang J, Lin Y, Zhang W. A fault diagnosis approach for rolling element bearings based on RSGWPT-LCD bilayer screening and extreme eearning eachine. IEEE Access 2017;5:5515-5530.

[36] Liu Y, Yang G, Li M, Yin H. Variational mode decomposition denoising combined the detrended fluctuation analysis. Signal Processing 2016;125:349-364.

[37] Li J, Zhang Y, Xie P. A new adaptive cascaded stochastic resonance method for impact features extraction in gear fault diagnosis. Measurement 2016;91:499-508.

[38] Li Z, Jiang Y, Wang X, Peng Z. Multi-mode separation and nonlinear feature extraction of hybrid gear failures in coal cutters using adaptive nonstationary vibration analysis. Nonlinear Dynamics 2016;84(1):295-310.

[39] Lei Y, Lin J, He Z, Zuo MJ. A review on empirical mode decomposition in fault diagnosis of rotating machinery. Mechanical Systems and Signal Processing 2013;35(1-2):108-126.

[40] Singh DS, Zhao Q. Pseudo-fault signal assisted EMD for fault detection and isolation in rotating machines. Mechanical Systems and Signal Processing 2016;81:202-218.

[41] Yang D, Liu Y, Li S, Li X, Ma L. Gear fault diagnosis based on support vector machine optimized by artificial bee colony algorithm. Mechanism and Machine 
Theory 2015;90:219-229.

[42] Chen Y, Kloft M, Yang Y, Li C, Li L. Mixed kernel based extreme learning machine for electric load forecasting. Neurocomputing 2018;312:90-106.

[43] Rajeswari C, Sathiyabhama B, Devendiran S, Manivannan K. A Gear fault identification using wavelet transform, rough set based GA, ANN and C4.5 algorithm. Procedia Engineering 2014;97:1831-1841.

[44] Deng W, Zhao H, Yang X, Xiong J, Sun M, Li B. Study on an improved adaptive PSO algorithm for solving multi-objective gate assignment. Applied Soft Computing 2017;59:288-302.

[45] Baig MZ, Aslam N, Shum HPH, Zhang L. Differential evolution algorithm as a tool for optimal feature subset selection in motor imagery EEG. Expert Systems with Applications 2017;90:184-195. 